\title{
In Vivo Dynamic Metabolic Changes After Transplantation of Induced Pluripotent Stem Cells for Ischemic Injury
}

\author{
Shuang $\mathrm{Wu}^{* 1-3}$, Yuankai Zhu*1-3, Hao Liu ${ }^{1-3}$, Ling Tang ${ }^{4,5}$, Ruili $\mathrm{Du}^{1-3}$, Yehua Shen ${ }^{1-3}$, Jin Feng ${ }^{1-3}$, Kai Zhang ${ }^{1-3}$, \\ Caiyun $\mathrm{Xu}^{1-3}$, Shouhong Zhang ${ }^{1-3}$, Yao Chen ${ }^{1-3}$, Fahuan Song ${ }^{1-3}$, Yunqi Zhu ${ }^{1-3}$, Weizhong Gu${ }^{6}$, Ping Liang ${ }^{4,5}$, \\ Ignasi Carrió ${ }^{7}$, Hong Zhang ${ }^{1-3}$, and Mei Tian ${ }^{1-3}$ \\ ${ }^{I}$ Department of Nuclear Medicine and Medical PET Center, Second Hospital of Zhejiang University School of Medicine, Hangzhou, \\ China; ${ }^{2}$ Institute of Nuclear Medicine and Molecular Imaging, Zhejiang University, Hangzhou, China; ${ }^{3}$ Key Laboratory of Medical \\ Molecular Imaging of Zhejiang Province, Hangzhou, China; ${ }^{4}$ First Affiliated Hospital, Zhejiang University School of Medicine, \\ Hangzhou, China; ${ }^{5}$ Institute of Translational Medicine, Zhejiang University, Hangzhou, China; ${ }^{6}$ Department of Pathology, Children's \\ Hospital of Zhejiang University School of Medicine, Hangzhou, China; and ${ }^{7}$ Nuclear Medicine Department, Hospital Sant Pau, \\ Autonomous University of Barcelona, Barcelona, Spain
}

\begin{abstract}
This study aimed to investigate in vivo dynamic metabolic changes after transplantation of induced pluripotent stem cells (iPSCs) and iPSC-derived enriched cardiomyocytes (iPSC-CMs) in a rat model of ischemic injury. Methods: Serial ${ }^{18} \mathrm{~F}-\mathrm{FDG} \mathrm{PET}$, echocardiographic, immunohistochemical, and immunofluorescence studies were performed after transplantation of iPSCs and iPSC-CMs and compared with embryonic stem cells (ESCs), ESC-CMs, and a phosphatebuffered saline control group of rats with myocardial infarction. Results: Increased glucose metabolism in periinfarct areas and improved myocardial function were observed in the stem cell transplantation groups compared with the control group, and serial immunofluorescence and immunohistochemical results exhibited the survival and migration of stem cells during the study period. Conclusion: Serial ${ }^{18}$ F-FDG PET and echocardiographic imaging studies demonstrated the dynamic metabolic changes and recovery of myocardial function after stem cell transplantation. ${ }^{18} \mathrm{~F}-\mathrm{FDG}$ PET could be a potential approach to evaluating spatiotemporal dynamic metabolic changes in vivo after transplantation of iPSCs or iPSC-CMs for ischemic injury.
\end{abstract}

Key Words: positron emission tomography (PET); metabolism; myocardial ischemia; induced pluripotent stem cell (iPSC); induced PSC-derived cardiomyocytes (iPSC-CMs)

J Nucl Med 2016; 57:2012-2015

DOI: 10.2967/jnumed.115.171124

$\mathbf{E}$ arly observations on the mechanisms of ischemic injury focused on relatively simple biochemical and physiologic changes known to result from interruption of circulation. Subsequent research has shown that molecular imaging has the potential ability to identify pathophysiologic changes in vivo, which is crucial for evaluating new therapeutic approaches to-

Received Dec. 10, 2015; revision accepted Aug. 3, 2016.

For correspondence or reprints contact: Mei Tian, Department of Nuclear Medicine, Second Hospital of Zhejiang University School of Medicine, 88 Jiefang Rd., Hangzhou, Zhejiang 310009, China.

E-mail: meitian@zju.edu.cn

${ }^{*}$ Contributed equally to this work.

Published online Aug. 25, 2016.

COPYRIGHT (c) 2016 by the Society of Nuclear Medicine and Molecular Imaging, Inc. ward ischemia injury. Recently, rapid progress in stem cell technologies has triggered increasing interest in the use of pluripotent stem cells-including embryonic stem cells (ESCs) $(1,2)$ and a new cell type, induced pluripotent stem cells (iPSCs) $(3,4)$ - in cardiovascular ischemic repair. iPSCs own many features similar to ESCs and can detour the risk of immune rejection and ethical issues. Although electrocardiographic studies have found that iPSC-derived cardiomyocytes (iPSC-CMs) might be an exciting cell source for renewing myocardium in vitro and improving cardiac function (5), a noninvasive, sensitive, repeatable, and quantitative imaging modality is imperative to better understand the in vivo behavior and efficacy of transplantation of iPSCs and iPSC-CMs.

Since PET can be used clinically for both cell trafficking and therapeutic response monitoring, it has been referred to as one of the best-suited modalities for evaluating the therapeutic effect of stem cells $(6,7)$. Thus, we hypothesized that ${ }^{18} \mathrm{~F}$-FDG PET might be useful for the in vivo evaluation of spatiotemporal dynamic metabolic changes after transplantation of iPSCs or iPSC-CMs. To verify our hypothesis, we performed ${ }^{18}$ F-FDG small-animal PET combined with echocardiography to evaluate metabolic and functional recovery after transplantation of iPSC and iPSC-CMs in a rat model of myocardial infarction, and we confirmed the findings using autoradiographic, immunohistochemical, and immunofluorescence analysis.

\section{MATERIALS AND METHODS}

\section{Animal Model and Stem Cell Administration}

An animal model of myocardial infarction was established by permanent ligation of the coronary artery (8). Enhanced green fluorescent protein-labeled mouse iPSCs (miPSCs) and mouse ECSs (mESCs), and $\mathrm{H} 9$ human ESCs (hESCs) and human iPSCs (hiPSCs), were cultured as described previously (9). hESCs and hiPSCs were differentiated into cardiomyocytes (hESC-CMs and hiPSC-CMs) using a 2-dimensional monolayer protocol and maintained in a $5 \% \mathrm{CO}_{2} /$ air environment. Details of the generation of stem cells and the cell culture conditions are provided in the supplemental materials (available at http://jnm.snmjournals.org).

To investigate metabolic changes after transplantation of iPSCs, we divided 39 adult male Sprague-Dawley rats into 3 groups (13 rats per group): a control group, an mESC group, and an miPSC group. 


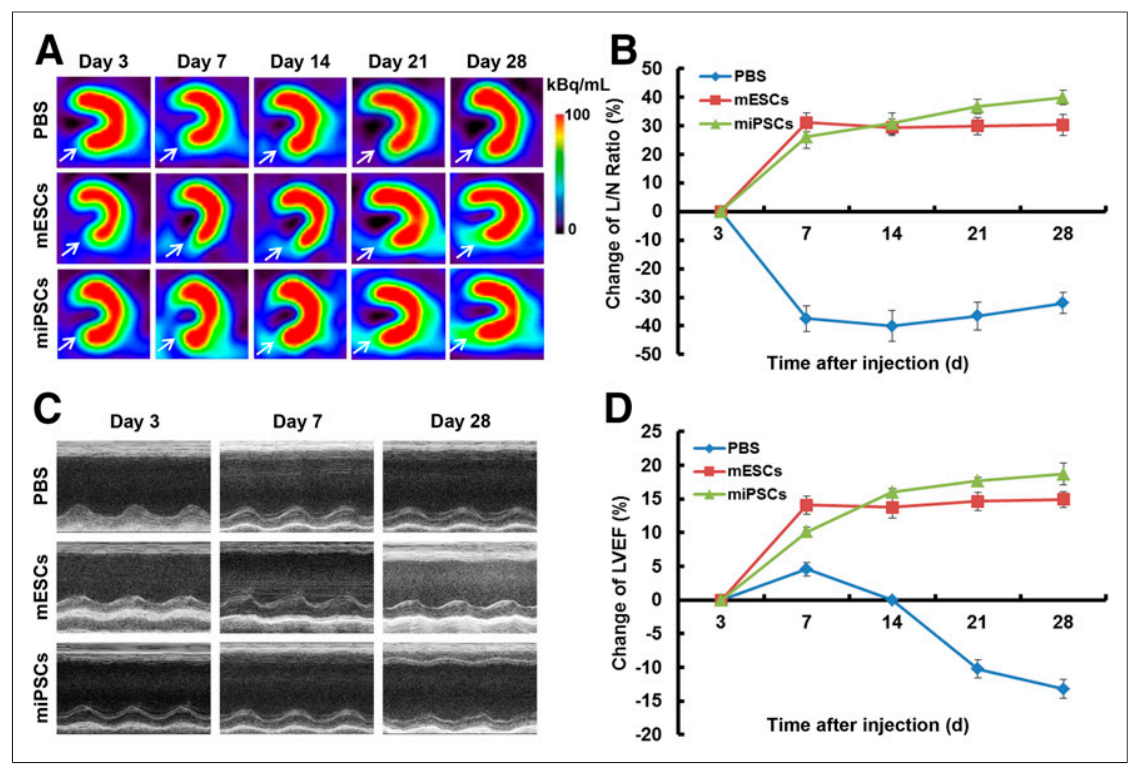

FIGURE 1. Serial PET and echocardiographic studies on rats after transplantation of miPSCs and mESCs compared with control rats in model of myocardial infarction. (A) Axial PET images demonstrating better metabolic recovery after stem cell transplantation (arrows). Scale is set according to signal intensity. (B) Semiquantitative analysis of change in $\mathrm{L} / \mathrm{N}$ ratio demonstrating significant increase in glucose metabolism in miPSC group and mESC group than in control group $(P<0.05)$. (C and D) M-mode echocardiographic images (C) and LVEF analysis (D) demonstrating significantly greater cardiac functional improvement in rats after transplantation of miPSCs and mESCs than in control rats $(P<0.05) . \mathrm{PBS}=$ phosphate-buffered saline.

Furthermore, to explore whether iPSC-CMs could improve the metabolic function of injured rodent heart, an additional 24 rats were divided into 3 groups ( 8 rats per group, all receiving tacrolimus daily): a control group, hESC-CM group, and hiPSC$\mathrm{CM}$ group. Thirty minutes after myocardial infarction, $2 \times 10^{6}$ cells were implanted by intramyocardial injection into the periinfarct area. Control animals received an equal volume of phosphate-buffered saline without cells (details are provided in the supplemental materials). The animal protocol was approved by the Institutional Animal Care and Use Committee of Zhejiang University School of Medicine (protocol ZJU201407-1-02-070).

\section{${ }^{18}$ F-FDG PET, Echocardiography, and Autoradiography}

Repeated ${ }^{18}$ F-FDG PET and echocardiographic studies were performed on days 3, 7, 14, 21, and 28 after stem cell transplantation. The lesion-to-normal tissue $(\mathrm{L} / \mathrm{N})$ ratio was calculated as (maximal counts per pixel of lesion region of interest)/(maximal counts per pixel of normal area) (10). Then, the change in $\mathrm{L} / \mathrm{N}$ ratio on day $\mathrm{N}$ was calculated as $[(\mathrm{L} / \mathrm{N}$ ratio on day $\mathrm{N}-\mathrm{L} / \mathrm{N}$ ratio on day $3) /(\mathrm{L} / \mathrm{N}$ ratio on day 3$)] \times 100 \%(n=3,7$, 14, 21, and 28). Transthoracic 2-dimensional and M-mode echocardiographic images were obtained at the level of the papillary muscle using a Vevo 2100 system (VisualSonics Inc.). Change in left ventricular ejection fraction (LVEF) on day $\mathrm{N}$ was calculated as [(LVEF on day $\mathrm{N}-$ LVEF on day 3)/(LVEF on day 3$)] \times 100 \%(n=3,7,14$, 21 , and 28). The autoradiographic imaging was performed after the last PET scan.

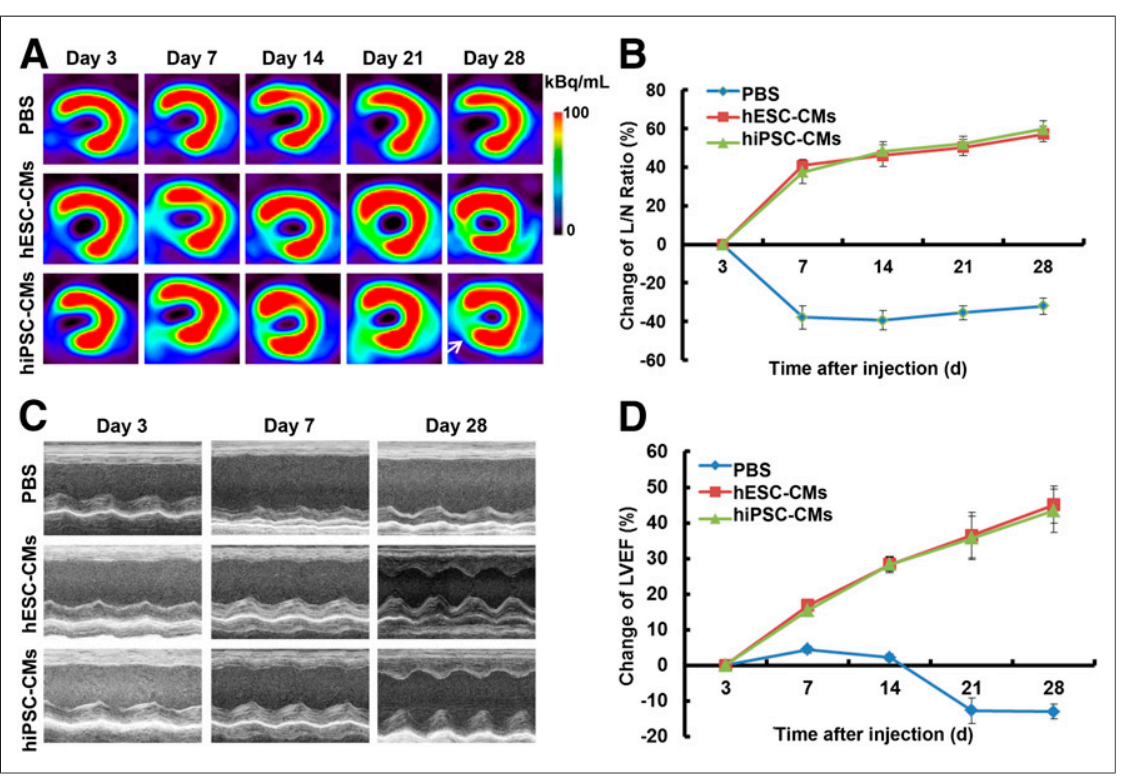

FIGURE 2. Serial PET and echocardiographic studies on rats after transplantation of hiPSC-CMs and hESC-CMs compared with control rats in model of myocardial infarction. (A) Axial PET images demonstrating better metabolic recovery after transplantation of hiPSC-CMs and hESC-CMs (arrows). Scale is set according to signal intensity. (B) Semiquantitative analysis of change in L/N ratio demonstrating significant increase in glucose metabolism in hiPSC-CM group and hESC-CM group than in control group $(P<0.05)$. (C and D) M-mode echocardiographic images $(C)$ and LVEF analysis (D) demonstrating significantly greater cardiac functional improvement in rats after transplantation of hiPSC-CMs and hESC-CMs than in control rats $(P<0.05)$. PBS $=$ phosphate-buffered saline.

\section{Immunohistochemical and} Immunofluorescence Analysis

Primary antibodies against von Willebrand factor (vWF), $\alpha$-smooth-muscle actin (SMA), cardiac troponin I (cTNI), and cardiac troponin $\mathrm{T}$ (cTNT) were used for immunohistochemical staining. Serial immunofluorescence analysis was processed for tracking the fate of enhanced green fluorescent protein-labeled transplanted cells on days $3,7,14,21$, and 28. Cardiomyocytes, microvessels, and arterioles were determined by cTNT, $v W F$, and $\alpha$-SMA, respectively.

\section{Statistical Analysis}

All data are presented as mean $\pm \mathrm{SD}$. Oneway ANOVA was performed to analyze group differences, and Pearson correlation was used to correlate changes in $\mathrm{L} / \mathrm{N}$ ratio with changes in LVEF. A $P$ value of less than 0.05 was considered to represent statistical significance.

\section{RESULTS}

\section{Imaging Findings}

PET imaging allowed visualization of ${ }^{18}$ F-FDG accumulation throughout the 28-d study (Figs. 1A and 2A).

From days 7 to 28 , the changes in glucose metabolism (shown as change in $\mathrm{L} / \mathrm{N}$ ratio 
in Figs. 1B and 2B) in the periinfarct area in both the mESC group $(P<0.05)$ and the miPSC group $(P<0.05)$ were significantly higher than those in the control group (Fig. 1B). Glucose metabolism increased gradually in the miPSC group until days $21(P<$ $0.05)$ and $28(P<0.05$; Fig. 1B), compared with the mESC group. Similarly, Figure 2B shows the glucose metabolism to increase significantly from days 7 to 28 in the hiPSC-CM group $(P<$ $0.05)$ and the hESC-CM group $(P<0.05)$, compared with the control group. However, no significant difference was found between the hESC-CM group and the hiPSC-CM group on days 7, 14,21 , or 28 ( $P=0.170,0.421,0.356$, and 0.199 , respectively).

Similarly, on echocardiographic images, myocardial function (shown as change in LVEF) improved significantly in the stem cell groups from days 7 to 28 after transplantation, compared with the control group (Figs. 1C and 2C). Compared with the mESC group, the change in LVEF was significantly lower in the miPSC group on day $7(P<0.05)$ but increased gradually and became significantly higher from days 14 to 28 ( $P<0.001$; Fig. 1D). No significant difference was found between the hESC-CM group and the hESCCM group on days $7,14,21$, or $28(P=0.087,0.994,0.801$, and 0.492, respectively; Fig. 2D).

The PET finding agreed with data generated from the autoradiographic studies. During the study period, no teratomas formed in the heart or other thoracic organs after transplantation of the stem cells or stem cell-derived cardiomyocytes.

\section{Immunohistochemical and Immunofluorescence Analysis}

Immunohistochemical staining demonstrated that the numbers of vWF-positive and integrated optical density $\alpha$-SMA-positive cells were significantly higher in the miPSC group and the hiPSC$\mathrm{CM}$ group than in the control group $(P<0.05)$; however, no statistically significant difference was found between the miPSC group and the mESC group or between the hiPSC-CM group and the hESC-CM group (Figs. 3A-3E). The integrated optical density of cTNI and cTNT in the miPSC group and the hiPSC-CM group was significantly higher than that in the control group $(P<0.05)$; however, no statistically significant difference was found between the miPSC group and the mESC group $(P=0.444)$ or between the hiPSC-CM group and the hESC-CM group $(P=0.688)$.

Serial immunofluorescence studies on miPSCs and mESCs observed more cTNT-positive cells within $21 \mathrm{~d}$ (on days 3 and 14-21) than on day 28 and more vWF-positive and $\alpha$-SMApositive cells within $14 \mathrm{~d}$ than after day 21 (Supplemental Figs. 1-5).

\section{DISCUSSION}

In this study, serial ${ }^{18}$ F-FDG PET combined with echocardiographic studies demonstrated increased glucose metabolism and improved myocardial function in stem cell transplantation rats than in control rats. Specially, there were two important findings. First, compared with the control group, a steadily increasing recovery of glucose metabolism and improvement of function was found after transplantation of iPSCs, hiPSC-CMs, and hESC-CMs. Second, serial immunofluorescence and immunohistochemical results exhibited survival and migration of stem cells within $28 \mathrm{~d}$. To the best of our knowledge, this is the first direct comparison between miPSCs and mESCs, as well as between hESC-CMs and hiPSC-CMs, in the same rat model of myocardial infarction using serial ${ }^{18} \mathrm{~F}-\mathrm{FDG}$ PET and echocardiographic studies, and this study identified longitudinal agreement between PET imaging and immunohistochemical/immunofluorescence studies after stem cell therapy.

Selection of a better pluripotent stem cell and a more feasible noninvasive imaging technique is essential for translating preclinical research to future clinical practice. In the past few years, most studies have focused on cell activity and behavior in vitro. Only a few studies have investigated in vivo trafficking or monitoring of transplanted stem cells in myocardial infarction. ESCs could be identified at 2 wk after transplantation on initial studies of fused ${ }^{18}$ F-FDG and $9-\left(4-{ }^{18}\right.$ F-fluoro3-(hydroxymethyl)butyl)guanine imaging (1), and the latter combined with MR imaging presented excellent detection of double labeling of ESCs with the HSV1-sr39tk reporter gene and superparamagnetic iron oxide particles for up to $4 \mathrm{wk}$ in myocardial infarction rats (2). Recently, several studies have reported that iPSC-CMs provide functional benefit after myocardial infarction, although there has been no consensus about the protective mechanism induced by these differentiated cardiomyocytes (11). By using cardiac MRI and comparing with a nontreated control, $\mathrm{Wu}$ et al. (12) found that iPSC-CMs promoted cardiac protection and attenuated cardiac remodeling after myocardial infarction, despite limited engraftment detected with bioluminescence imaging. In our present study, we found that the iPSC group and the iPSC-CM group showed a steady increase in ${ }^{18} \mathrm{~F}-\mathrm{FDG}$ accumulation during the 4-wk period whereas the ESC
FIGURE 3. Immunohistochemical staining of a-SMA, vWF, cTNI, and cTNT (arrows). (A) Images of ischemic region in miPSC rats, mESC rats, and control rats. (B) Images of ischemic region in hiPSC-CM rats, hESC-CM rats, and control rat. Scale bar $=50 \mu \mathrm{m}$. (C) a-SMA-positive cells. ${ }^{*} P<$ 0.05 , compared with control. (D) vWF-positive cells. ${ }^{*} P<0.05$, compared with control. (E) cTNIand cTNT-positive cells. ${ }^{*} P<0.05$, compared with control. IOD = integrated optical density; PBS = phosphate-buffered saline.
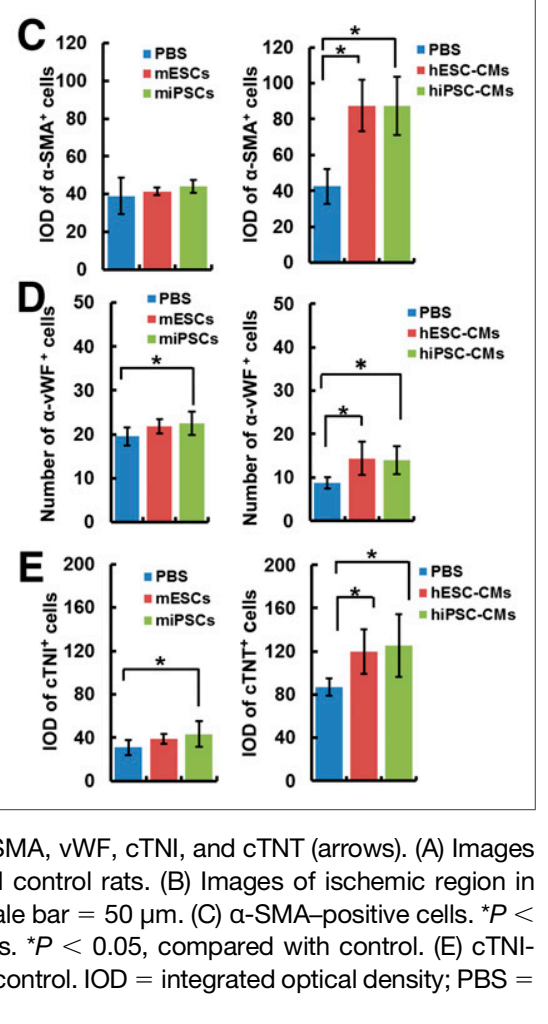
group showed a higher accumulation only in the earlier stage, indicating that iPSCs, especially iPSC-CMs, may produce a more stable therapeutic response than ESCs or ESC-CMs.

Furthermore, in our serial immunofluorescence analysis (with 5 time points), transplanted stem cells survived, migrated to the periinfarct area, and expressed the markers of myocardial cells (cTNI and cTNT), endothelial cells (vWF), and smooth muscle or myofibroblast cells ( $\alpha$-SMA). This observation suggests that metabolic and functional recovery after stem cell transplantation might be due to transplanted cells differentiating into myocardial cells, secreting paracrine factors, or recruiting peripheral stem cells to the ischemic territory (11). Interestingly, a very recent study (13) found that treatment with hESC-derived myocardial precursors improved cardiac function (with a smaller infarct and less wall thinning), induced endogenous angiogenesis (with increased capillary density), and reduced apoptosis of host cardiomyocytes at the periinfarct area within 4 wk after myocardial infarction in mice (13). Unfortunately, that study performed staining at only 2 time points. In contrast, our serial immunofluorescent staining allowed us to examine the full timecourse after post-myocardial infarction stem cell transplantation and revealed that more cells were cTNT-positive, vWF-positive, and $\alpha$-SMA-positive at the earlier stages than at the later ones.

\section{CONCLUSION}

Serial ${ }^{18}$ F-FDG PET and echocardiographic imaging studies demonstrated the dynamic metabolic changes and myocardial functional recovery after stem cell transplantation. ${ }^{18} \mathrm{~F}$-FDG PET could be a potential approach to the in vivo evaluation of spatiotemporal dynamic metabolic changes after transplantation of iPSCs or iPSC-CMs for ischemic injury.

\section{DISCLOSURE}

The costs of publication of this article were defrayed in part by the payment of page charges. Therefore, and solely to indicate this fact, this article is hereby marked "advertisement" in accordance with 18 USC section 1734. This work was supported by grants from the National Science Foundation of China (NSFC) (81170306), Science Technology Program of Zhejiang Province (2012C13013-2), and National Major Research Program of China (2016YFA0100900) and by the Fundamental Research Funds for the Central Universities of
China (2013QNA7021). No other potential conflict of interest relevant to this article was reported.

\section{ACKNOWLEDGMENTS}

We thank Drs. Zhi Jiang and Han Chen for their excellent technical support.

\section{REFERENCES}

1. Cao F, Lin S, Xie X, et al. In vivo visualization of embryonic stem cell survival, proliferation, and migration after cardiac delivery. Circulation. 2006;113:10051014.

2. Qiao H, Zhang H, Zheng $\mathrm{Y}$, et al. Embryonic stem cell grafting in normal and infarcted myocardium: serial assessment with MR imaging and PET dual detection. Radiology. 2009;250:821-829.

3. Carpenter L, Carr C, Yang CT, et al. Efficient differentiation of human induced pluripotent stem cells generates cardiac cells that provide protection following myocardial infarction in the rat. Stem Cells Dev. 2012;21:977-986.

4. Lang C, Lehner S, Todica A, et al. Positron emission tomography based in-vivo imaging of early phase stem cell retention after intramyocardial delivery in the mouse model. Eur J Nucl Med Mol Imaging. 2013;40:1730-1738.

5. Kawamura M, Miyagawa S, Miki K, et al. Feasibility, safety, and therapeutic efficacy of human induced pluripotent stem cell-derived cardiomyocyte sheets in a porcine ischemic cardiomyopathy model. Circulation. 2012;126(11, suppl 1)S29-S37.

6. Zhang Y, Ruel M, Beanlands RS, et al. Tracking stem cell therapy in the myocardium: applications of positron emission tomography. Curr Pharm Des. 2008;14:3835-3853.

7. Jiang $\mathrm{H}$, Cheng Z, Tian M, Zhang $\mathrm{H}$. In vivo imaging of embryonic stem cell therapy. Eur J Nucl Med Mol Imaging. 2011;38:774-784.

8. Masumoto H, Matsuo T, Yamamizu K, et al. Pluripotent stem cell-engineered cell sheets reassembled with defined cardiovascular populations ameliorate reduction in infarct heart function through cardiomyocyte-mediated neovascularization. Stem Cells. 2012;30:1196-1205.

9. Takahashi K, Yamanaka S. Induction of pluripotent stem cells from mouse embryonic and adult fibroblast cultures by defined factors. Cell. 2006;126:663676.

10. Perin EC, Tian M, Marini FC, et al. Imaging long-term fate of intramyocardially implanted mesenchymal stem cells in a porcine myocardial infarction model. PLoS One. 2011;6:e22949.

11. Lalit PA, Hei DJ, Raval AN, Kamp TJ. Induced pluripotent stem cells for postmyocardial infarction repair: remarkable opportunities and challenges. Circ Res. 2014;114:1328-1345.

12. Ong SG, Huber BC, Hee Lee W, et al. Microfluidic single-cell analysis of transplanted human induced pluripotent stem cell-derived cardiomyocytes after acute myocardial infarction. Circulation. 2015;132:762-771.

13. Ye J, Gaur M, Zhang Y, et al. Treatment with hESC-derived myocardial precursors improves cardiac function after a myocardial infarction. PLoS One. 2015;10:e131123. 